\title{
An Extender Kalman Filter-Based Induction Machines Faults Detection
}

\author{
Abdelghani Chahmi*, Mokhtar Bendjebbar*, Bertrand Raison**, Mohamed Benbouzid*** \\ *Electrical Drives Laboratory LDEE, University of Science and Technology of Oran, USTO/MB.Oran, Algeria \\ ** Grenoble Electrical Engineering Laboratory, G2Elab \\ $* * *$ University of Brest, LBMS
}

\section{Article Info}

Article history:

Received Nov 9, 2015

Revised Dec 16, 2015

Accepted Jan 5, 2016

\section{Keyword:}

Extended Kalman filter

Induction motor

Modeling

Rotor defect

Stator defect

\begin{abstract}
This paper deals with the detection and localization of electrical drives faults, especially those containing induction machines. First, the context of the study is presented and an Extended Kalman Filter is described for induction machines fault detection. Then the modeling procedure under faculty conditions is shown, and the machine diagnosis methods are developed. The proposed diagnosis approach requires only little experimental data, and more importantly it provides efficient simulation tools that allow characterizing faulty behavior. Fault detection uses signal processing techniques in known operating phases (fixed speed), considering and locating malfunctions.
\end{abstract}

Copyright $(2016$ Institute of Advanced Engineering and Science. All rights reserved.

\section{Corresponding Author:}

Abdelghani Chahmi, Electrical Drives Laboratory LDEE, University of Science and Technology of Oran USTO/MB

El Mnaouer, BP 1505, Bir El Djir, Oran, Algeria 31000

Email: chahmi.abdelghani@gmail.com

\section{INTRODUCTION}

Large industrial systems are widely confronted to the challenges of reliability and availability of the production devices. Electric drives containing an induction machine are largely used in the industrial applications thanks to their low costs, high performances and robustness [1]. As known, a system can realize the assigned task, only under conditions ensuring the security. An early detection of anomalies in electrical motors may help avoiding downtimes [2]. Thus, in order to detect an incipient fault, we should give a special attention to the spectral analysis of stator currents [3], [4]. Most of recent researches on defects detection of induction machines use the stator currents analysis. Several condition-monitoring methods [5] have been developed and were used to help the detection of electric defects in induction machine. Among these methods, the authors in [6] consider the vibrations monitoring, torque control, the temperature monitoring, etc.

In this paper, the rotor resistance is estimated using a method of Extended Kalman Filter (EKF). This state observer is proved to be a useful tool to determine the parameter variations [7], [8]. In this paper, it is shown that EKF can be employed to identify the disturbance in rotor resistance. Recently, the Kalman filter was applied successfully for defect forecasting in [9]. For example it is used to interpolate the trends of a signal-learned $g$ from the recorded data and then estimate the evolution of defects in the proposed diagnosis methods in [10]. In this study, the proposed approach considers the value of rotor resistance as fixed for condition monitoring. This value in the diagnostic tools which one uses is not fixed contrary to the classical approaches of control of machine. Hence, we propose a method that considers a variation of rotor resistance in order to give a good estimate as well as a good robustness for the control of the induction machine. 
This paper is organized as follows: part II describes the induction machine model under simplifying assumptions. Induction machine models have been developed under healthy and faulty conditions in the (abc) reference frame. Part III, presents a state of the art review of Extended Kalman Filter. Finally, part IV gives digital simulations of the proposed faulty model. The obtained results prove the effectiveness of the proposed monitoring method based on an Extended Kalman Filter.

\section{INDUCTION MOTOR DRIVES}

\subsection{Overview of the Drive}

The drive under study is composed of a machine with induction, inverter of width of impulse, ordering of rotor flow - directed, a loop of measurement of the current and a loop of speed control. Figure 1 presents the total diagram of the unit.

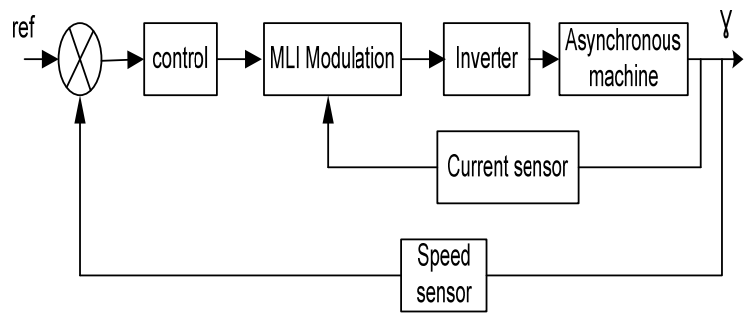

Figure 1. Schematic diagram of the system under study

\subsection{Model of the Induction Motor}

The objective of this section is to present the different models used for our study. This is first of all to model the induction machine finely enough to introduce a relevant modeling of defects. Then we present how the default templates are not used in their simplified and balanced version diagnostic purposes.

\subsubsection{Models Simulation Proposed in the Reference Frame (abc)}

The induction machine is a known system-well [11]; models exist and allowing, in the majority of the cases and under certain simplifying assumptions, a representation of the machine without fault. These assumptions are the following ones [12-13]:

- The air-gap is of constant width without effect of notch (not of eccentricity, of unbalance, reduction amongst harmonics in the development of inductances expression).

- Assimilation of the cage to a short-circuit having the same number of phases as static winding (simplification of the equations of the machine by taking into account the average effect of the cage).

- Sinusoidal distribution, along the air-gap, of the magnetic fields of each winding (simplification of the equations of the machine by taking into account of the expressions in complex of the currents, allows to use a vectorial model).

- Absence of saturation in the magnetic circuit (identical behavior of each phase, allows use of a vectorial model).

- The influences of the effect of skin and the heating of the drivers are neglected (not variation of the parameters intervening in the machine under normal functioning).

- Star coupling of windings (the currents form a system balanced under normal functioning).

Under the conditions pointed out previously, the equations of the electrical circuits utilize clean and mutual inductances making it possible to define flows according to the currents [12].

On the level of the stator, the tensions check:

$$
\left[\begin{array}{l}
V s a \\
V s b \\
V s c
\end{array}\right]=\left[R_{s}\right]\left[\begin{array}{l}
i s a \\
i s b \\
i s c
\end{array}\right]+\frac{d}{d t}\left[\begin{array}{l}
\varphi s a \\
\varphi s b \\
\varphi s c
\end{array}\right]
$$

Where the resistances matrix is written according to the supposition of symmetry: 


$$
\left[R_{s}\right]=\left[\begin{array}{ccc}
r_{s} & 0 & 0 \\
0 & r_{s} & 0 \\
0 & 0 & r_{s}
\end{array}\right]
$$

On the level of the rotor, under - assumption-that - rotor-is comparable to a rotor with three-phase rollings up, the voltage are written:

$$
\left[\begin{array}{l}
V r a \\
V r b \\
V r c
\end{array}\right]=\left[R_{R}\right]\left[\begin{array}{c}
i r a \\
i r b \\
i r c
\end{array}\right]+\frac{d}{d t}\left[\begin{array}{c}
\varphi r a \\
\varphi r b \\
\varphi r c
\end{array}\right]
$$

Matrix $R_{R}$ has the same form that $R_{S}$, flux vector are expressed according to the inductances matrix and stator and rotor currents.

$$
\begin{aligned}
& {\left[\begin{array}{l}
\varphi s a \\
\varphi s b \\
\varphi s c
\end{array}\right]=\left[L_{s}\right]\left[\begin{array}{l}
i s a \\
i s b \\
i s c
\end{array}\right]+\left[M_{S R}\right]\left[\begin{array}{l}
i r a \\
i r b \\
i r c
\end{array}\right]} \\
& \left.\left[\begin{array}{l}
\varphi r a \\
\varphi r b \\
\varphi r c
\end{array}\right]=\left[M_{R S}\right]\left[\begin{array}{l}
i s a \\
i s b \\
i s c
\end{array}\right]+\left[L_{R}\right]\right]\left[\begin{array}{l}
i r a \\
i r b \\
i r c
\end{array}\right]
\end{aligned}
$$

In order to be able to give an account of the interactions between the phases, we expressed each resistance and inductance matrix according to the number of whorls of rollings up of the machine.

$$
\begin{aligned}
& {\left[R_{S}\right]=\left[\begin{array}{ccc}
R_{s a} & 0 & 0 \\
0 & R_{s b} & 0 \\
0 & 0 & R_{s c}
\end{array}\right]=R_{s}\left[\begin{array}{ccc}
n_{s a} & 0 & 0 \\
0 & n_{s b} & 0 \\
0 & 0 & n_{s c}
\end{array}\right]} \\
& {\left[R_{R}\right]=\left[\begin{array}{ccc}
R_{r a} & 0 & 0 \\
0 & R_{r b} & 0 \\
0 & 0 & R_{r c}
\end{array}\right]=R_{r}\left[\begin{array}{ccc}
n_{r a} & 0 & 0 \\
0 & n_{r b} & 0 \\
0 & 0 & n_{r c}
\end{array}\right]}
\end{aligned}
$$

nsa corresponds to the number of whorls of the phase a has stator.

$$
\begin{aligned}
& {\left[L_{S}\right]=\left[\begin{array}{ccc}
L_{s a} & M_{s a b} & M_{s c a} \\
M_{s a b} & L_{s b} & M_{s b c} \\
M_{s c a} & M_{s b c} & L_{s c}
\end{array}\right]=\left[\begin{array}{ccc}
n_{s a}^{2} l_{s} & n_{s a} n_{s b} m_{s} & n_{s a} n_{s c} m_{s} \\
n_{s a} n_{s b} m_{s} & n_{s b}^{2} l_{s} & n_{s b} n_{s c} m_{s} \\
n_{s a} n_{s c} m_{s} & n_{s b} n_{s c} m_{s} & n_{s c}^{2} l_{s}
\end{array}\right]} \\
& {\left[L_{R}\right]=\left[\begin{array}{ccc}
L_{r a} & M_{r a b} & M_{r c a} \\
M_{r a b} & L_{r b} & M_{r b c} \\
M_{r c a} & M_{r b c} & L_{r c}
\end{array}\right]=\left[\begin{array}{ccc}
n_{r a}^{2} l_{r} & n_{r a} n_{r b} m_{r} & n_{r a} n_{r c} m_{r} \\
n_{r a} n_{r b} m_{r} & n_{r b}^{2} l_{r} & n_{r b} n_{r c} m_{r} \\
n_{r a} n_{r c} m_{r} & n_{r b} n_{r c} m_{r} & n_{r c}^{2} l_{r}
\end{array}\right]} \\
& {\left[M_{S R}(\theta)\right]=\left[\begin{array}{ccc}
n_{s a} n_{r a} m_{s r} \cos (\theta) & n_{s a} n_{r b} m_{s r} \cos (2 \pi / 3) & n_{s a} n_{r c} m_{s r} \cos (\theta-2 \pi / 3) \\
n_{s b} n_{r a} m_{s r} \cos (\theta-2 \pi / 3) & n_{s b} n_{r b} m_{s r} \cos (\theta) & n_{s b} n_{r c} m_{s r} \cos (\theta+2 \pi / 3) \\
n_{s c} n_{r a} m_{s r} \cos (\theta+2 \pi / 3 & n_{s c} n_{r b} m_{s r} \cos (\theta-2 \pi / 3) & n_{s c} n_{r c} m_{s r} \cos (\theta)
\end{array}\right]} \\
& {\left[M_{S R}(\theta)\right]=\left[M_{R S}(\theta)\right]^{T}}
\end{aligned}
$$




\subsubsection{Cage Electric Failures in the (abc) Model}

This model that we have just described in the previous part allows in particular creating asymmetrical faults in both stator and rotor by the variation amongst whorl of the rolling up of the phase at fault. The defect can be localized on one or more phases.

It should be noted that this modeling can be criticized: the defects of type short-circuit are represented in the model like removals of whorls i.e. openings partial of phase; that does not correspond completely to reality since it would also be necessary to take account of the shading rings [13]. This model constitutes a progress compared to the balanced models or /and considering only one variation of resistance usually used to validate the algorithms [14].

\subsection{Model “The Good Performance” for Control and of Monitoring}

Models are necessary to design and improve the controllers and the observers. They are commonly balanced. One passes from the reference frame (abc), fixes, with the fixed reference frame $(\alpha, \beta)$ by the transformation of Concordia [15].

$$
\left\{\begin{array}{l}
p X=A_{c} X+B_{c} U \\
Y=C_{c} X
\end{array}\right.
$$

With $\quad \mathrm{X}=\left[\begin{array}{lll}i_{s \alpha} & i_{s \beta} \varphi_{r \alpha} \varphi_{r \beta}\end{array}\right]^{\mathrm{T}}$

$$
\begin{aligned}
& A_{c}=\left[\begin{array}{cccc}
-\gamma & 0 & \frac{\mathrm{K}}{\tau} & \mathrm{K} \omega \\
0 & -\gamma & -K \omega & \frac{\mathrm{K}}{\tau} \\
\frac{L_{m}}{\tau} & 0 & -\frac{1}{\tau} & -\omega \\
0 & \frac{L_{m}}{\tau} & \omega & -\frac{1}{\tau}
\end{array}\right] B_{c}=\left[\begin{array}{cccc}
\frac{1}{\sigma L_{s}} & 0 & 0 & 0 \\
0 & \frac{1}{\sigma L_{s}} & 0 & 0
\end{array}\right]^{T} \quad C_{c}=\left[\begin{array}{llll}
1 & 0 & 0 & 0 \\
0 & 1 & 0 & 0
\end{array}\right] \\
& \text { and } \quad \gamma=\frac{R_{s}}{\sigma L_{s}}+\frac{1-\sigma}{\sigma \tau} \tau=\frac{L_{r}}{R_{r}} \mathrm{~K}=\frac{L_{m}}{\sigma L_{s} L_{r}} \sigma=1-\frac{L_{m}^{2}}{L_{s} L_{r}}
\end{aligned}
$$

\subsection{Induction Machine Discrete Model}

We develop in this paragraph a discrete model of an induction machine in order to be able to use these equations in the development of Kalman Filter. We consider indeed that the equations of the observer at ends of diagnosis will be inevitably used in a system containing microprocessor or other and thus entered in the form of a discrete model.

The discrete version is obtained by using the formula of Euler:

$$
\left\{\begin{array}{l}
\frac{x(k+1)-x(k)}{T_{s}}=A_{c} x(k)+B_{c} U(k) \\
y(k)=C_{c} x(k)
\end{array}\right.
$$

$$
\begin{array}{ll}
\text { Of or } & \left\{\begin{array}{l}
x(k+1)=\left(T_{s} A_{c}+I\right) x(k)+T_{s} B_{c} U(k) \\
y(k)=C_{c} x(k)
\end{array}\right. \\
\text { Or } \quad\left\{\begin{array}{l}
x(k+1)=A_{d} x(k)+B_{d} U(k) \\
y(k)=C_{d} x(k)
\end{array}\right. \\
A_{d}=I+A_{c} T_{s}, B_{d}=B_{c} T_{s}, C_{d}=C_{c}
\end{array}
$$

\subsection{Induction Machine Observer-Based EKF}

This part presents the proposed method to supervise the application. We will develop the method by using the observer model previously described. 


\subsubsection{The Extended Kalman Filter}

The basic equations of the extended Kalman filter are deduced from that of the discrete model of the machine in good fonctionnement.la discrete matrix of state $A_{d}=I+A_{c} T_{s}$. One introduces noises of state $w(k)$ and noises of measurement $v(k)$ [16] - [17]. The function $\mathrm{F}$ is defined so that one to extend filter of Kalman has all the parameters of the machine. The estimate of rotor resistance is considered like means of monitoring.

$$
\left\{\begin{array}{l}
x(k+1)=A_{d} x(k)+B_{d} U(k)+w(k) \\
y(k)=C_{d} x(k)+v(k)
\end{array}\right.
$$

Where

$$
f(x(k), U(k))=A_{d} x(k)+B_{d} U(k)
$$

The process noise $\mathrm{w}(\mathrm{k})$ is characterized by

$$
\begin{aligned}
& E\{w(k)\}=0 \\
& E\left\{w(k) w(k)^{T}\right\}=Q
\end{aligned}
$$

Where $\mathrm{Q}$ is definite positive.

The measurement noise $\mathrm{v}(\mathrm{k})$ respects:

$$
\begin{aligned}
& E\{v(k)\}=0 \\
& E\left\{v(k) v(k)^{T}\right\}=R
\end{aligned}
$$

$\mathrm{R}$ is definite positive too.

If we call $\mathrm{P}$ the matrix of covariance of the error in estimation, this matrix is obtained to leave:

$$
p(k / k)=E\left\{(x(k)-\hat{x}(k)) \cdot(x(k)-\hat{x}(k))^{T}\right\}
$$

The notation $\mathrm{k} / \mathrm{k}$ corresponds to a prediction at time $\mathrm{K}$ based on data up to and including time $\mathrm{K}$ (a priori covariance) and $\mathrm{k}+1 / \mathrm{k}$ corresponds to a prediction at time $\mathrm{k}+1$ starting from the data until the time $\mathrm{K}$ (a posteriori covariance). In the following equations, $t$ represents the Jacobian matrix of the nonlinear function $\mathrm{f}$ is used [7].

$$
J=\left.\frac{\partial f}{\partial x(k)}\right|_{\hat{x}(k), U(k)}
$$

Applied to the system under study, J can be expressed as

$$
J=\left[\begin{array}{ccccc}
1-\gamma T_{s} & 0 & \frac{\mathrm{K}}{\tau} T_{s} & K \omega T_{s} & -\frac{1}{\sigma L_{s}} T_{s} \hat{i}_{s d} \\
0 & 1-\gamma T_{s} & -\mathrm{K} \omega T_{s} & \frac{\mathrm{K}}{\tau} T_{s} & -\frac{1}{\sigma L_{s}} T_{s} \hat{i}_{s q} \\
\frac{L_{m}}{\tau} T_{s} & 0 & 1-\frac{T_{s}}{\tau} & -\omega T_{s} & 0 \\
0 & \frac{L_{m}}{\tau} T_{s} & \omega T_{s} & 1-\frac{T_{s}}{\tau} & 0 \\
0 & 0 & 0 & 0 & 1
\end{array}\right]
$$

At each integration time, the Kalman Filter equations to be solved are:

$1 /$ Computation of the observer states

$$
\hat{x}(k+1 / k)=J \hat{x}(k / k)+B_{d} U(k)
$$


2/ Computation of the a posteriori covariance matrix $\mathrm{P}(\mathrm{k}+1 / \mathrm{k})$

$$
P(k+1 / k)=J P(k / k) J^{T}+Q
$$

3/ Computation of the outputs of the filter

$$
\begin{aligned}
& \hat{x}(k+1 / k+1)=\hat{x}(k+1 / k)+G_{d}(k+1) . \\
& {\left[y(k+1)-C_{d} \hat{x}(k+1 / k)\right]}
\end{aligned}
$$

4/ Computation of the feedback gain matrix of the Kalman Filter

$$
\begin{gathered}
G_{d}(k+1)=P(k+1 / k) C_{d}(k+1)^{T} \\
{\left[C_{d}(k) P(k+1 / k) C_{d}(k)^{T}+R\right]^{-1}}
\end{gathered}
$$

5/ Computation of the a priori covariance matrix $\mathrm{P}(\mathrm{k}+1 / \mathrm{k}+1)$

$$
\begin{aligned}
& P(k+1 / k+1)=P(k+1 / k)- \\
& G_{d}(k+1) C_{d}(k+1) P(k+1 / k)
\end{aligned}
$$

We consider that $\mathrm{Q}$ and $\mathrm{R}$ matrix are constant but they can be time variant. The behavior and the stability of the Kalman Filter are functions of $\mathrm{Q}$ and $\mathrm{R}$.

\section{RESULTS AND ANALYSIS}

The test conditions are the following:

- From $\mathrm{t}=0$ to $\mathrm{t}=0.3 \mathrm{~s}$, flux application

- From 0.3 to $1.8 \mathrm{~s}$, liner increase of the speed reference

- From 1.8s constant speed

- At $\mathrm{t}=3 \mathrm{~s}$, fault in the machine

The observers are enabled from beginning.

\subsection{Good Performance}

Our approach in this part of simulation is to see the behavior of the induction machine in healthy mode and with electric defect (rotor, stator, inverter) in the mode transitory and permanent, after from there will introduce an observer on the outlet side of the machine (extended Kalman filter) is to see its reaction. The results result from rotor resistance, the stator current FFT.

The following figure takes the form of the current in a phase of the induction machine. One can distinguish there the various phases related to the order from the system: phase of fluxing, phase of application of the instruction of couple related to the slope of speed, then stabilization the speed and instruction of couple. Up to 0.3 second, the purpose of the applied voltages is to position the vector flow in the machine, as from this moment, an instruction of couple is applied to the order and speed believes.

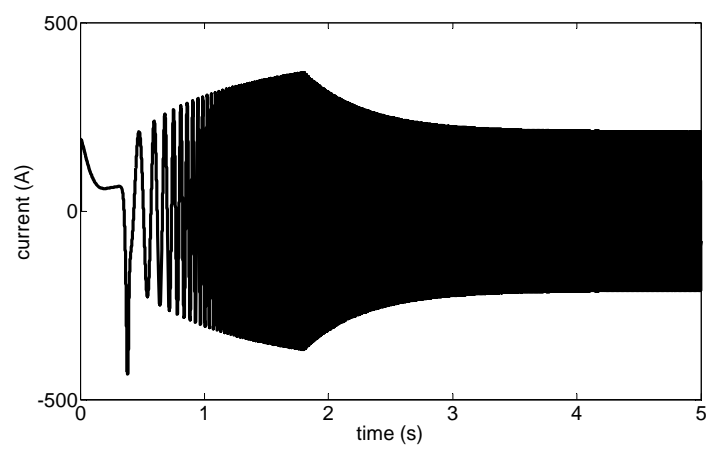

Figure 2. Stator current 
The following figure takes the form of stator flux following the axis alpha. One finds well the phase of fluxing as well as the evolution of flow according to the increase number of revolutions of the machine and thus of the frequency of the supply voltages.

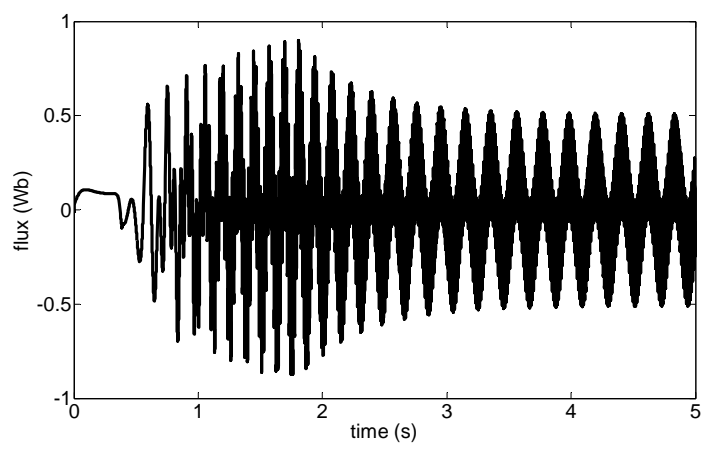

Figure 3. Stator flux

The following figure presents the evolution of the produced torque by the induction machine. One finds the phase of acceleration (with a going beyond related to the speed control) and the phase of stabilization speed and thus couple.

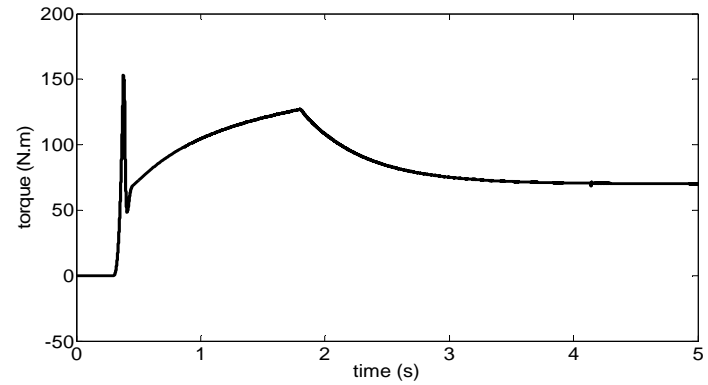

Figure 4. Machine couples

The following figure takes the form the speed of the system. One finds well there the various phases, which one describes previously.

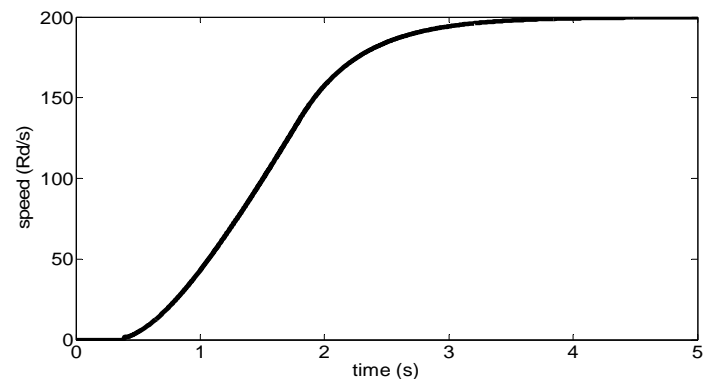

Figure 5. Speed of the machine

The following figure takes the form of rotor resistance at exit of the filter of Kalman (for a healthy system). One notes a drift of rotor resistance according to speed. This derivative was corrected: according to 
speed, the estimated parameter is compensated for so that it is closer to the expected value. This compensation has the form of a linear relation between the drift of rotor resistance and speed.

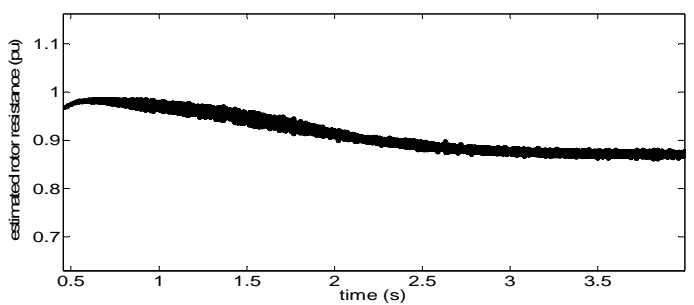

Figure 6. Estimate of rotor resistance by the filter of Kalman without compensation

speed

The following figure takes the form of rotor resistance with compensation of the drift according to

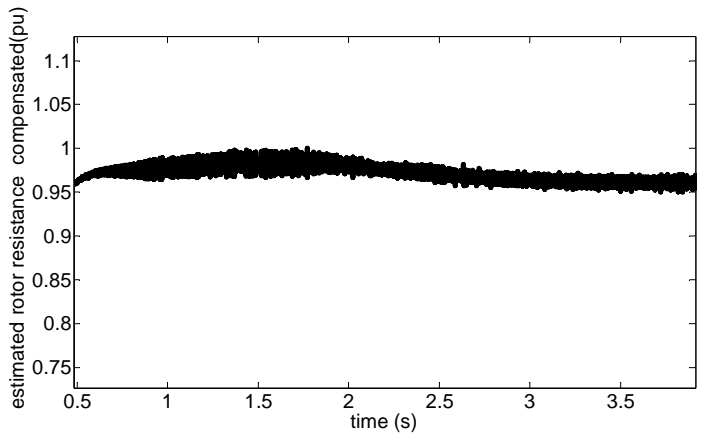

Figure 7. Estimated rotor resistance by the Kalman filter with compensation

\subsection{Rotor Faults}

Figure 8 takes the form of the stator current for an unbalanced defect $(40 \%$ of whorls removed in the phase at fault), note the appearance of the lines in the spectrum of the current. The defect with the rotor is characterized by the appearance of two frequencies $(1 \pm 2 \mathrm{~g})$ or $\mathrm{g}$ represents the slip $(92,2 \mathrm{~Hz}$ et $107 \mathrm{~Hz})$ around the electric frequency f electric $(100 \mathrm{~Hz})$. If the FFT of this signal is made, this defect will be very difficult to detect in the spectrum of the current because the amplitude of the line at the fundamental frequency is very important compared to that of the required line and these lines are at a frequency frame the line with fundamental with a difference related to the slip (in general very weak).

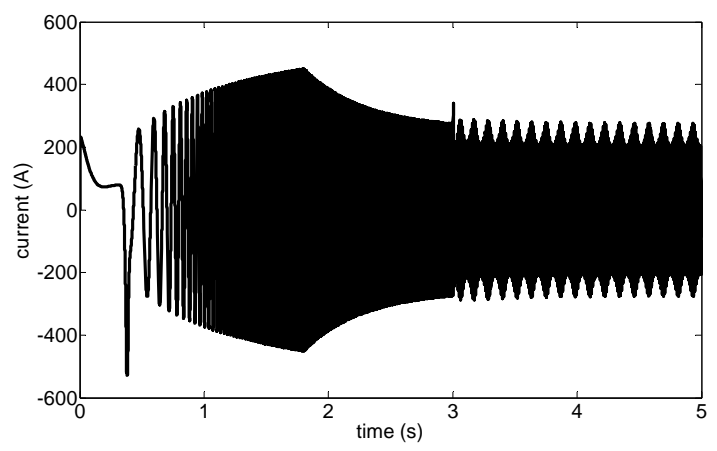

Figure 8. Stator current under rotor fault 
The following figure takes the form of resistance estimated for a defect unbalanced to the rotor ( $40 \%$ of whorls removed at fault on a phase). Noting however that the estimate of rotor resistance is assigned to the moment $\mathrm{t}=3$ second (time of the application of defect) from where appearance of the low frequency oscillations. This existence of the frequency allows then on the basis of resistance rotor estimated characterizing that it is well a defect with the rotor.

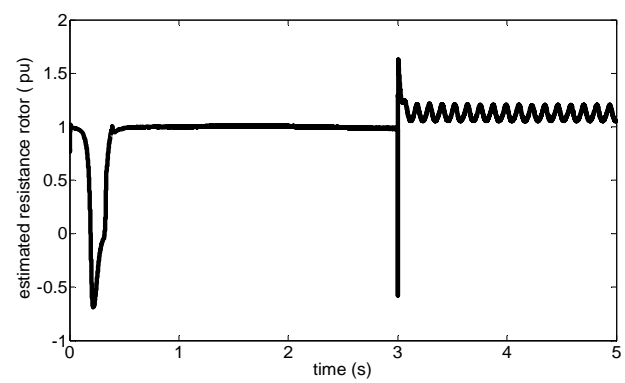

Figure 9. Estimation resistance rotor for an asymmetrical fault witch the rotor

\subsection{Defect with the Stator}

Figure 10 takes the form of the stator current for a defect unbalanced to the stator $(40 \%$ of whorls removed at fault on a phase). Let us note the appearance of the frequency, which characterizes the defect, which is 3 felectric $(300 \mathrm{~Hz})$. The appearance of the lines in the spectrum of the current is due to the application of the defect to the moment $t=3 \mathrm{~s}$, it is easy to detect the frequency, which characterizes the defect in low frequency. (See figure below after the stator phase current FFT).

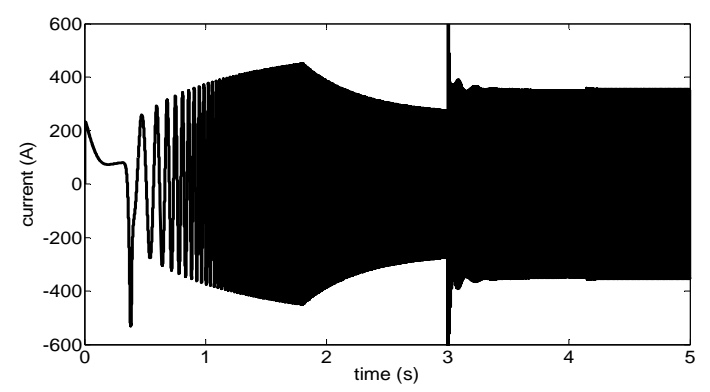

Figure 10. Stator current of the machine at fault to the stator

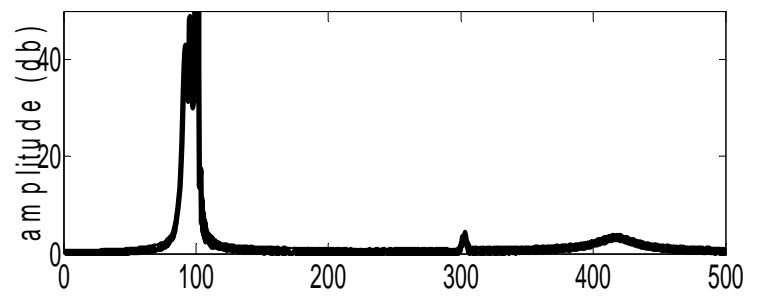

Figure 11. FFT of the static current of the phase

The following figure presents the current of the phase at exit of the machine and the current for the same phase at exit of the observer, it should be specified that the two currents (measured and estimated) are similar. The observer thus modified the rotor size resistance so that the two currents are "identical". The error, which exists between the two currents, explains by the fact why the observer is a discrete system: he thus does the calculations of the sizes on the basis of measure taken at previous time. This explains the light error of hauling. 

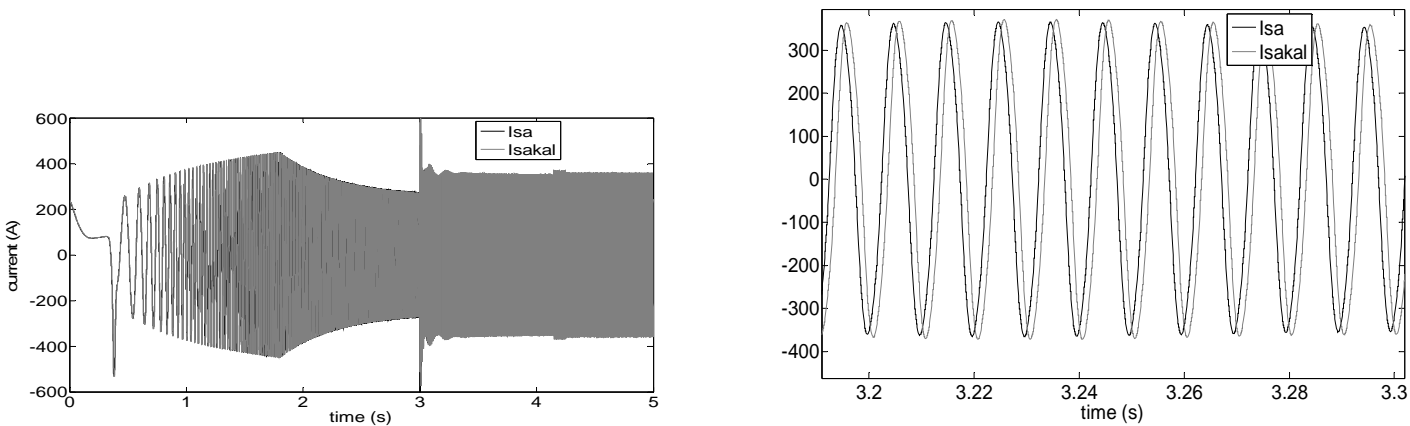

Figure 12. Phase current (Isa), Observed current (Isakal)

Figure 12 illustrates the application of this estimator on the exit of the machine leads to the convergence of resistance up to 3 second (application of the defect). It is noted whereas the observer does not manage to make converge the resistance estimated following the application of the defect. This is explained because the model used for the equations of the observer is a balanced model. However the defect results in an unbalanced behavior of the machine. The observer has of another choice to only make evolve dynamically rotor resistance so that the other states are coherent compared to measurements.

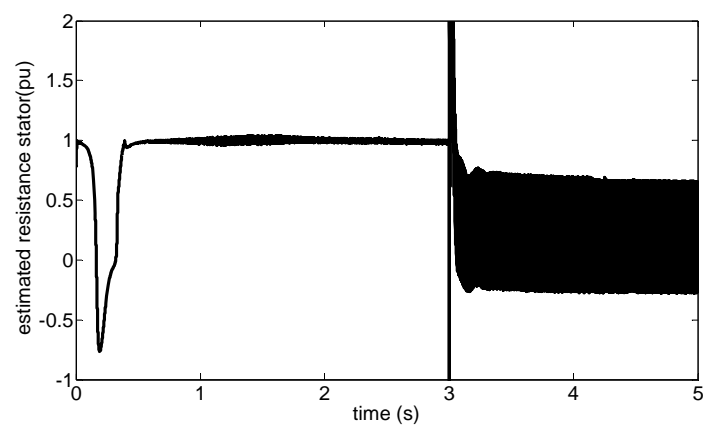

Figure 13. Estimation rotor resistance for an asymmetrical fault witch the stator

\subsection{Synchronous Demodulation Applied to Rotor Resistance}

Each defect has a frequency which characterizes it, the idea of this method consists with research the amplitude of the line associated with this frequency to detect the evolution of this one. The principle of this demodulation is exposed to the following figure.

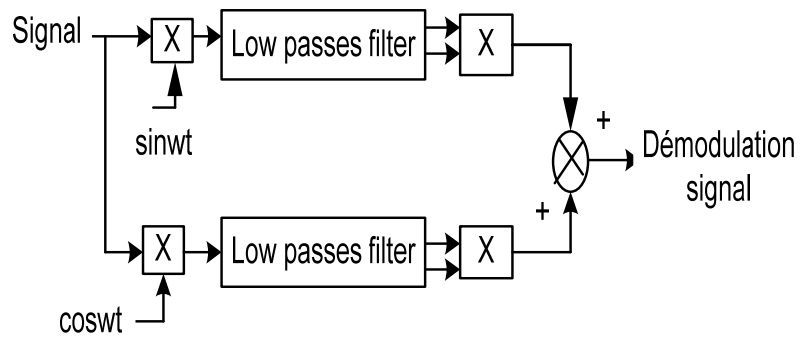

Figure 14. Principe of the demodulation

The following figure has the amplitude of the line associates with the frequency of defect ( $2 \mathrm{fe})$. The characteristic is well detected by this monitoring system. The defect is applied to 3 seconds. 


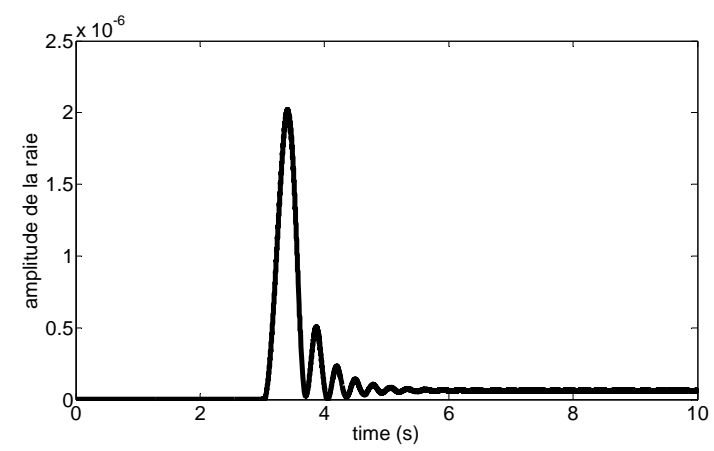

Figure 15. Compensated resistance rotor demodulated

\subsection{Inverter Fault}

Figure 16 takes the form of the stator current of the machine at fault of inverter. We chose to give an account of the effects of an open component. For that, we included a block allowing to block the control commands arriving at the switch considered as failing. After the period of fluxing $(0 \mathrm{~s}-0.3 \mathrm{~s})$, one notices that the size is sinusoidal until the application of the defect to the moment $t=3$ seconde. The occurrence of the defect thus leads to the appearance of the frequencies, which characterize this defect in low and high frequency (see figure 17 which presents the FFT of the stator current).

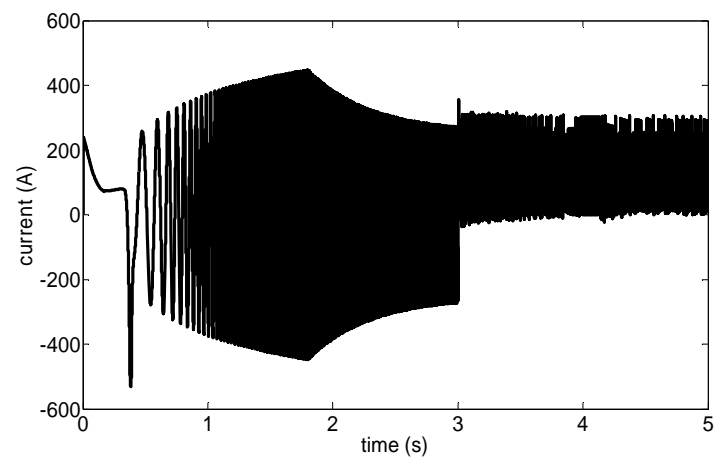

Figure 16. Stator current of the machine at fault of inverter
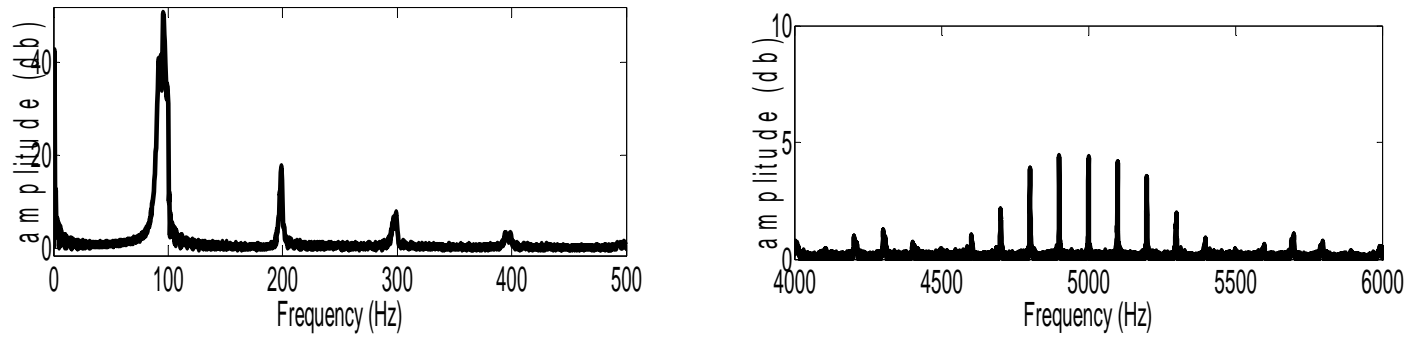

Figure 17. FFT of the current in low and high frequency

\subsubsection{La Demodulation Synchronous Applied to Rotor Resistance}

The following figure was obtained by using the demodulation of rotor resistance. One notes well the appearance of the frequency related to the MLI in estimated resistance. 


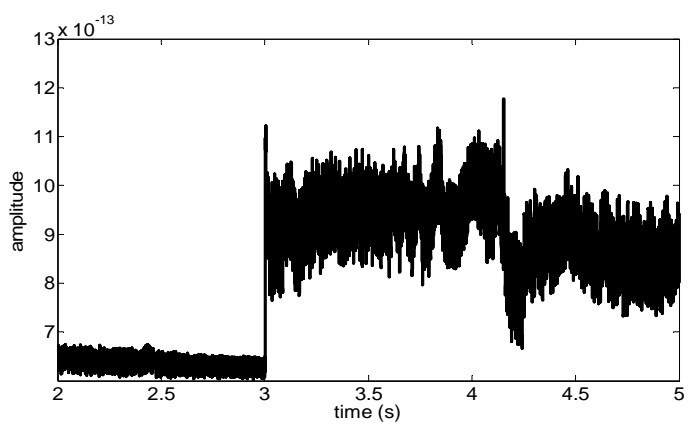

Figure 18. Compensated rotor resistance demodulated

Figure 19 illustrates the application of this estimator on the exit of the machine leads to the convergence of resistance up to 3 second (application of the defect). It is noted whereas the observer does not manage to make converge the resistance estimated following the application of the defect. The frequencies, which exist then, are as for the other defects related to the manifestation of the defect. It can then be useful to follow the existence of the frequency related to the PWM. This frequency does not exist under normal functioning is easy to detect because of its fixed value (contrary to the frequencies characteristic of the stator and rotor faults which are related on the stator frequency and speed).

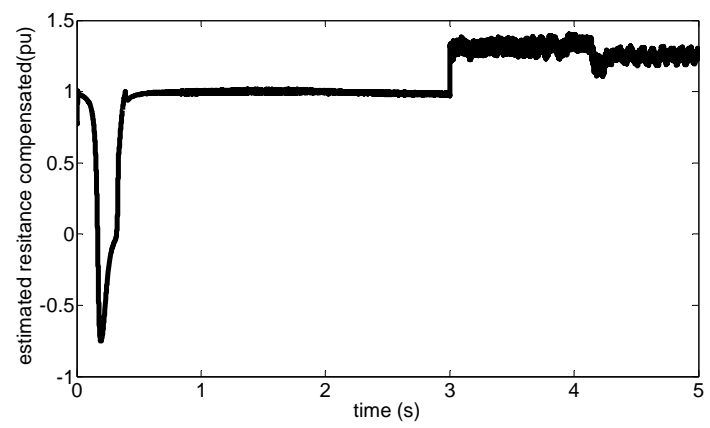

Figure 19. Estimation resistance rotor of the machine for a defect of inverter

\section{CONCLUSION}

The study presented in this paper is a contribution to the diagnosis of the defects of electric machinery, with special focus on squirrel-cage induction machine. Indeed, induction machines are appropriate for applications where high power and speed are required. This is due, to multiple advantages (simplicity of construction, robustness, performances...). However, for certain applications requiring high degree of reliability, it is necessary to carry out a fast and early detection of possible failures. The presented study focus on the contribution of the analytical in this article exposes the contribution of the analytical method containing models of the system to the diagnosis of the electric defects of the asynchronous machines. The vectorial control of the induction machine requires observers (possibly extended). We first of all showed that the use of an extended observer for diagnosis purpose made it possible to detect the principal defects in the electrical machine.

The parameter used for the diagnosis is the estimated rotor resistance. We showed that by coupling a spectral analysis of the estimated parameter that the technique could appear useful. The Kalman filter uses dynamics of the rotor resistance, which defines the evolution its value in time domain to obtain reliable data in the case of healthy machine (convergence of the estimate). We are aware of having studied only certain aspects of a very vast subject of study, which requires the comprehension of the system and the use of suitable tools.

The prospects with this work are multiple, from the point of view of the monitoring, the use of other parameters (stator resistance, stator inductance) to evaluate the performance of the wide observer. Then, from the point of view the detection of the defects, it will be interesting to study the monitoring of this kind of machine used as a generator of power (of wind turbines type for example) connected to the electrical. 


\section{REFERENCES}

[1] A. Chahmi, M. Bendjebbar, B. Raison. Fault Detection In Electrical Drives - Approach Signal. Electrical Sciences and Technologies in Maghreb CISTEM International Conference On, Tunis, Tunisia. 2014.

[2] M. Ferradj, N. Benouzza, A. Bendiabdellah, B. Mazari. Synthesis of Electrical Quantities Applied to Squirrel Cage Induction Motor Bearing Faults Detection.International Review of Electrical Enginnering (IREE). 2013; 8(5): 1446 -1460 .

[3] A.H. Boudinar, A. Bendiabdellah, N. Bennouza, N. Boughanemi. Three Phase Induction Motor Incipient Rotor's Faults Detection Based on Improved Root-MUSIC Approach. International Review of Electrical Engineering (IREE). 2007; 2(3): 406- 413.

[4] A. Lebaroud and G. Clerc. Classification of induction machine faults by optimal time-frequency representations. IEEE Transactions Industrial Electronics. 2008; 55(12): 4290-4298.

[5] E.H. Bouchikhi, V. Choqueuse, M.E Benbouzid., J.A. Antonino-Daviu. Stator Current Demodulation for Induction Machine Rotor Faults Diagnosis. Green Energy, International Conference On. Sfax, Tunisia. 2014.

[6] A. Bellini, F. Filippetti, C. Tassoni, and G.A. Capolino. Advances In Diagnostic Techniques for Induction Machines. IEEE Transactions Onv Industrial Electronics. 2008; 55 (12) : 4109-4126.

[7] D. Atkinson, J. Finch, P. Acarnley. Estimation of Rotor Resistance in Induction. IEE Proc-Electric. Power Appl.1996; 143(1); 87-94.

[8] A.J. Fernandez Gomez, V.H Jaramillo, J.R Ottewill. Fault Detection in Electric Motors by Means of the Extended Kalman Filter as Disturbance Estimator. Control UKACC International Conference On. 2014; Loughborough, Royaume-Uni.

[9] P. Lall, J. Wei, and K. Goebel. Comparison of Lalman-Filter and Extended Kalman-Filter for Prognostics Health Management of Electronics. In Proc; IEEE ITherm. 2012: 1281-1291.

[10] R.K Singleton, E.G Strangas, S. Aviyente. Extended Kalman Filtering for Remaining-Useful-Life Estimation of Bearings. Industrial Electronics, Journal IEEE Transactions On. 2015; 62(3): 1781-1790.

[11] P. Alger. The Nature of Induction Machine. (Gorden and Breach Science Publishers, 1965).

[12] W.Leonhard. Control Of Electrical Drives. (Springer Verlag, 1985).

[13] J.P. Caron, J.P. Hautier . Modélisation et Commande de la Machine Asynchrone. (Technip).

[14] R. Fiser, S. Ferkolj. Modelling of Dynamic Performance of Induction Machine with Rotor Faults. 1996; ICEM Proceedings 1;17-22.

[15] G.C. Verghese. Observers for Flux Estimation In Induction Machines. IEEE Trans. On Ind. Elec. 1988; 35( 1): 8591.

[16] D.J. Atkinson, P.P. Acarnley, J.W. Finch, Observers for Induction Motor State and Parameter Estimation”, IEEE Trans. On Id. 1991; 27 (6) : 1119-1127.

[17] B. Raison, F. Francois, G. Rostaing, J.P Rognon.Induction Drive Speed Monitoring by Neural Networks, Industrial Electronics Society. IECON, 26th Annual Conference of the IEEE. 2000; Nagoya, Japan.

\section{BIOGRAPHIES OF AUTHORS}
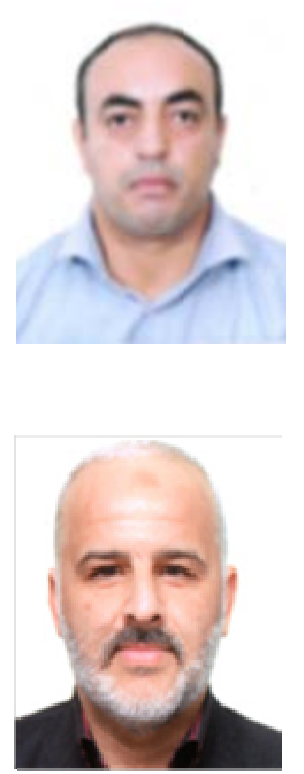

Abdelghani Chahmi was born in Mascara, Algeria, in 1975. He received the B.Sc. and M.Sc. degrees in electrical engineering, in 1999 and 2006, respectively, from the Polytechnic Military School, Algiers and the University of Sciences and Technology of Oran, Algeria. He is currently pursuing Ph.D. studies at the University of Sciences and Technology Mohamed Boudiaf, Oran, Algeria. Mr Chahmi is member in the Electric drives of Development Laboratory (LDEE). His current research interests fault diagnosis of electric machines.

Mokhtar Bendjebbar was born on August, 1965 in Relizane Algeria. He received his B.S degree in Electrical Engineering from the University of Sciences and Technology of Oran, Algeria, in1989, and the M.S degree from the same University of Sciences and Technology of Oran, in 1993. He is currently Professor of Electrical Engineering at the University of Sciences and Technology of Oran. His research interests include Electrical machines and Drives Control, Power Electronics, as well as Intelligent Control. 


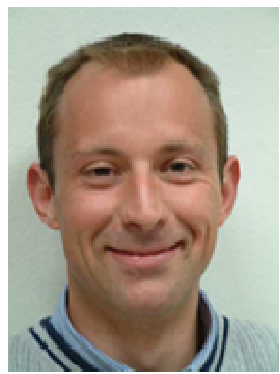

Bertrand Raison was born in Bethune, France, in 1972. He received the M.S. and Ph.D. degrees in electrical engineering from the Grenoble Institute of Technology, Grenoble, France, in 1996 and 2000, where he was an Associate Professor from 2001 to 2009. Since 2009; he has been a Professor at the ScienceUniversity Joseph Fourier, Grenoble. His general re-search interests include fault detection and localization in electrical systems and distribution network planning and protection with respect to fault management.

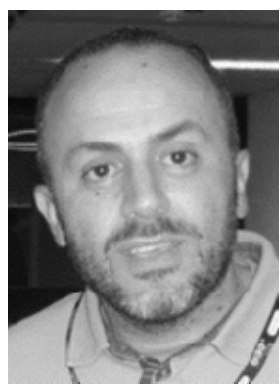

Mohamed El Hachemi Benbouzid was born in Batna, Algeria, in 1968. He received the B.Sc. degree in electrical engineering from the University of Batna, Batna, Algeria, in 1990, the M.Sc. and Ph.D. degrees in electrical and computer engineering from the National Polytechnic Institute of Grenoble, Grenoble, France, in 1991 and 1994, respectively, and the Habilitation à Diriger des Recherches degree from the University of Picardie "Jules Verne," Amiens, France, in 2000.After receiving the Ph.D. degree, he joined the Professional Institute of Amiens, University of Picardie "Jules Verne," where he was an Associate Professor of electrical and computer engineering. Since September 2004, he has been with the Institut Universitaire de Technologie of Brest, University of Brest, Brest, France, where he is a Professor of electrical engineering. His main research interests and experience include analysis, design, and control of electric machines, variable-speed drives for traction, propulsion, and renewable energy applications, and fault diagnosis of electric machines.

Prof. Benbouzid is an IEEE Senior Member. He is the Editor-in-Chief of the International Journal on Energy Conversion (IRECON). He is also an Associate Editor of the IEEE Transactions on Energy Conversion, the IEEE Transactions on Industrial Electronics, the IEEE Transactions on Sustainable Energy, and the IEEE Transactions on Vehicular Technology. He was an Associate Editor of the IEEE/ASME Transactions on Mechatronics from 2006 to 2009. 